\title{
Novel Method of Chairside Non-Bonded Fixed Retainer
}

\author{
Hayder Abdalla Hashim
}

\section{ABSTRACT}

Retention is the final phase of orthodontic treatment. It is defined as the 'stabilization of teeth in optimal esthetics and functional occlusion. A retention phase is considered crucial for the stability of treatment outcomes. The novel technique of utilizing a Non bonded fixed retainer (NFR) proved superior to the traditional bonded retainer in preventing relapse during the critical period spanning two to nine months after orthodontic therapy. The method as described in this article is simple to execute and economical. It makes effective plaque control in the vicinity of the retainer significantly easier. Moreover, this technique is recommended in patients with compromised periodontium. Nevertheless, it is essential for maintaining periodontal health and ensuring a successful outcome to treatment. However, studies on long term stability following lengthy periods of retention are needed. These are likely to substantiate the efficacy and durability of this technique.

Keywords: Fixed retainer, periodontal health, relapse, retention, stability.

Published Online: January 24, 2022

ISSN: $2684-4443$

DOI : $10.24018 /$ ejdent.2022.3.1.150

\section{H. A. Hashim*}

Department of Dentistry, Hamad

Medical Corporation, Hamad Dental Centre, Doha, Qatar.

(e-mail: hahashim78@yahoo.com)

*Corresponding Author

\section{INTRODUCTION}

Retention is the final phase of orthodontic treatment. It is defined as the 'stabilization of teeth in optimal esthetics and functional occlusion' [1]. A retention phase is considered crucial for the stability of treatment outcomes. An orthodontic retainer may be either fixed or removable, depending upon the nature of the malocclusion, the severity of the condition, and personal preference on the part of the clinician. Different designs of retainers have been used clinically, each with its own advantages and limitations. Fixed retainers can be either prefabricated or custom made to fit a specific patient. There are two approaches to the use of such appliances. The first involves bonding thicker stainless-steel wire of 0.032-inch gauge to the canines only. The use of nickel-titanium wire has also been reported [2]. The second method utilizes wire of 0.0175-0.0215-inch gauge bonded to each tooth from canine to canine in both upper and lower arches, or onto the lower incisor segment alone. The indications for each of these two approaches differ [1].

Fixed retainers are generally indicated when a lengthy period of retention is planned, or when the alignment achieved is unstable and patient compliance is poor. Zachrisson advocated the use of fixed bonded retention spanning all incisor teeth for cases of median diastema closure and following treatment in adult patients with compromised periodontal status [3]. Lee recommended retention limited to canines only in cases of severe rotation and crowding in the lower incisor region, deep overbite, patients whose treatment involved mandibular incisor proclamation, and changes in mandibular inter-canine width [4]. Tweed highlighted the correlation between upright incisors and enhanced stability and retention [5]. Along the same lines, Mills advocated accepting the existing position of lower incisors, given that they lie in a very narrow zone of stability [6], [7]. In keeping with this approach, Williams promoted the treatment objective of placing the lower incisors within $2 \mathrm{~mm}$ of the A-Pog line, a position he designated as being stable and esthetically optimal [8].

In contrast, Houston and coworkers stated that "...there is no evidence to support the use of a particular cephalometric line as a guide to posttreatment stability of the lower labial segment. Despite this, the use of such guides, that of the APog line, has become so widespread that the subject merits further examination. Though all the results were clinically stable; the A-Pog line was not found to be a reliable guide to lower incisor position [9]. What comprises stability and relapse differ between authors. Some consider the return of the lower incisors to their original positions as relapse.6,7 Others relate it to the degree of crowding or spacing measured in the lower incisor segment on casts and from cephalometric analyses carried out in the years following termination of treatment [10], [11].

Reference [12] introduced the technique and highlighted the advantages of applying multi-stranded wires as bonded retainers. This was followed by [13] applying this technique but limiting the bonding to canine teeth only. In a later publication [12] discussed the background leading to the evolution of multistrand fixed retainers along with his recommendations arising from more than twenty years of clinical experience with the technique. Included in his recommendations was the use of a 5-stranded 0.0215 -in gauge wire [14]. Reference [15] introduced a direct technique for fixed bonded retention that can be performed chair side immediately after debonding, and without the services of a dental technician. This approach can be used for both semi- 
permanent and permanent retention and has the advantages of ease of fabrication, superior adaptation to tooth surfaces, shorter treatment times and less demanding of patient compliance.

Upon following treated cases with fixed bonded canine to canine retention for a minimum of twenty years (and for as long as twenty-nine years), [16] concluded that permanent retention is an effective way of stabilizing mandibular incisor tooth position. It was found to be feasible to maintain effective hygiene and sound periodontal health in the presence of such fixed retention. The incidence of fracture in the retaining structure was low, and insufficient to negate its usage [16]. Recent years have seen the application of Computer Aided Design and Computer Aided Manufacture (CAD-CAM) applied to the fabrication of bonded retainers. There is little in the literature that reports on this technique. The Suresmile retainer (OraMetrix, Richardson, TX, USA) utilizing copper-nickel-titanium wire is one such example [17], as is the Memotain retainer (CA-Digital, Mettmann, Germany) manufactured from nickel titanium wire of 0.014x0.014-inch gauge [18].

Fixed retainers have the primary advantage of avoiding the placement and removal of the appliance by the patient, thus ensuring stabilization of the teeth in situ, and allowing for unhindered function. Their location on the lingual surfaces of teeth and the absence of any impact upon phonetics means that they are esthetic and avoid self-consciousness on the part of the patient. The strength of the bond to the teeth ensures the absence of any tooth movement, and stability of the alignment. Fixed retainers have the drawback of requiring more time for the patient in the dental chair than their removable counterparts do. Plaque control surrounding the appliance is more challenging as well. Furthermore, wires may break and possibly detach from the retainer, going unnoticed by the patient on occasion, and necessitating an intraoral repair to the appliance. This article aims to present a novel method of stabilization of the incisor teeth making use of soft preformed long ligature $<800 \mathrm{~N} / \mathrm{mm} 2$ Stainless Steel wire avoiding fixation with bonded composite resin (a nonbonded fixed retainer.

\section{MATERIAL AND MethoD}

Placement of a Non bonded fixed retainer requires use of the following which should be prepared in advance: Stainless steel long ligature wire, ligature cutters, condenser, tucker, mouth mirror, explorer, tweezers, ultrasonic scaler, mosquito forceps, contra angle hand-piece, rubber cup, and pumice.

\section{A. Method}

\section{1) Step 1: Debonding}

a) Following de-bracketing, debride the teeth of any composite remnants.

b) Polish all tooth surfaces with a rubber cup and pumice.

c) Using the ultrasonic scaler, eliminate any plaque or calculus deposits, especially in the mandibular incisor region.

d) Polish, wash, and use compressed air to dry the lingual and labial surfaces of the lower incisors which will be used to fix the retainer.

\section{2) Step 2: Fixation of the retainer}

i. Using preform Kobayashi ligature long soft $<800$ $\mathrm{N} / \mathrm{mm} 20.30 \mathrm{~mm} / 12$ Stainless Steel wire (Dentarum, Germany) commence at the lower left canine and separate the long ligature branches, passing one branch lingually and the other labially.

ii. Insert the labial branch of wire inter-proximally beneath the contact point of the lower left canine and first premolar to emerge on the lingual side, and in a similar manner insert the lingual branch to emerge on the labial side. Tighten both branches (Twice if possible) after tucking the wires beneath the gingival margin to hide them from sight.

iii. Repeat the same sequence with the lower left lateral and central incisors, and with the lower right central and lateral incisors, ensuring that the wire is tucked into the gingival sulcus on the labial aspect, (and if possible, on the lingual as well) with the help of a condenser

iv. Once the lower right canine is reached, reinsert the labial branch of the wire to emerge on the lingual side, and ensure once again that the labial wire is tucked into the gingival sulcus on all teeth. Holding both ends in mosquito forceps, twist them together and cut the wires with a ligature cutter, leaving approximately $3 \mathrm{~mm}$ of excess wire. Tuck this excess wire into the sulcus.

v. The patient is instructed in oral hygiene measures to ensure effective plaque control. Dental hygienists are alerted to the risk of damaging the wire, and the need for added caution when scaling is carried out.

\section{RESUlTS}

This patient sought treatment of severe crowding in lower incisors segment. Fixed orthodontic appliance with $0.022 \times 0.025 \mathrm{MBT}$ system was used. Upon debonding, the sequence detailed above was followed with the aim of stabilizing the outcome of treatment. Six months later, at a follow up appointment, all lower incisors remained well aligned and stable. The patient reported satisfaction with the outcome and able to maintain effective oral hygiene using a soft bristle toothbrush and dental floss without the need for a floss threader. The patient had not been instructed to avoid hard foods which typically pose a risk to conventional retainers. The same approach was applied to the second patient which exhibited stability during the first three critical months' post treatment. However, it is recommended to retighten the Figure eight wire retainer in the first and second follow up visits as well as stripping from canine to canine in case of any resistance during flossing to achieve unimpeded passage of floss between the teeth. 


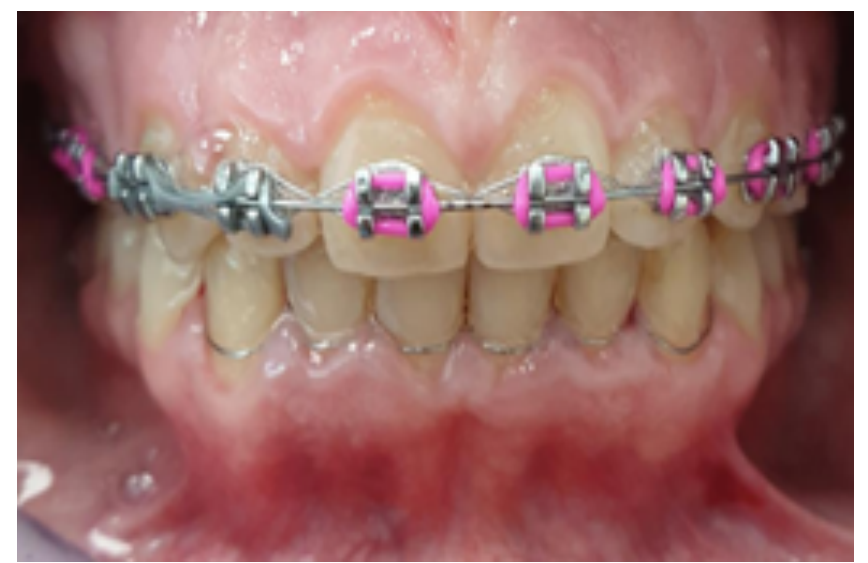

Fig. 1. Patient Lower arch front view at the time of debonding with nonbonded fixed retainer.

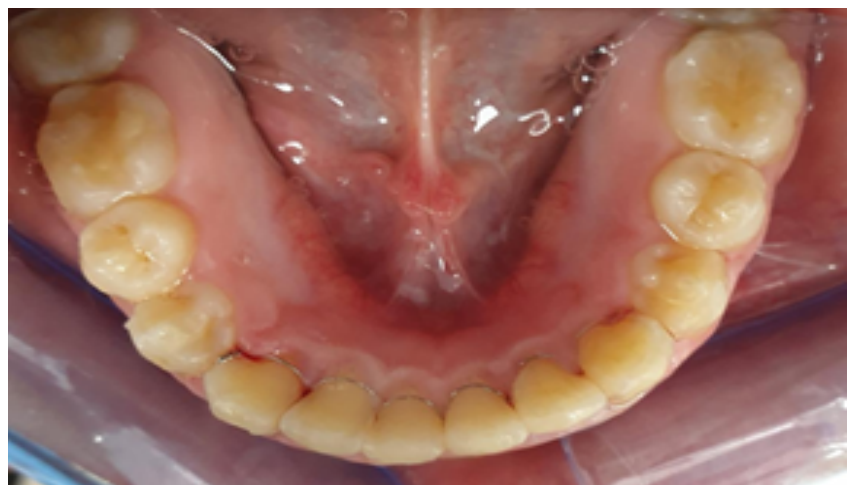

Fig. 2. Patient Occlusal view of lower arch at the time of debonding with non-bonded fixed retainer from 33 to 43 .

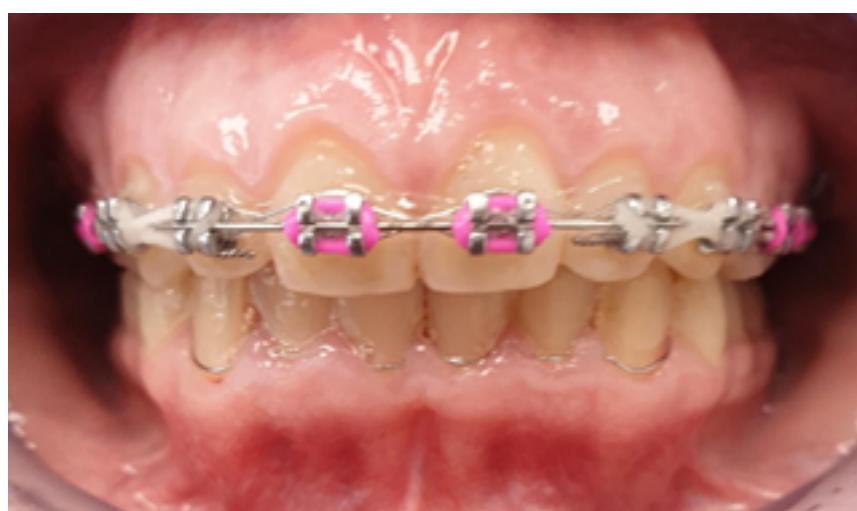

Fig. 3. Front view of lower arch one month after debonding with nonbonded fixed retainer from 33 to 43 (18 October 2020).

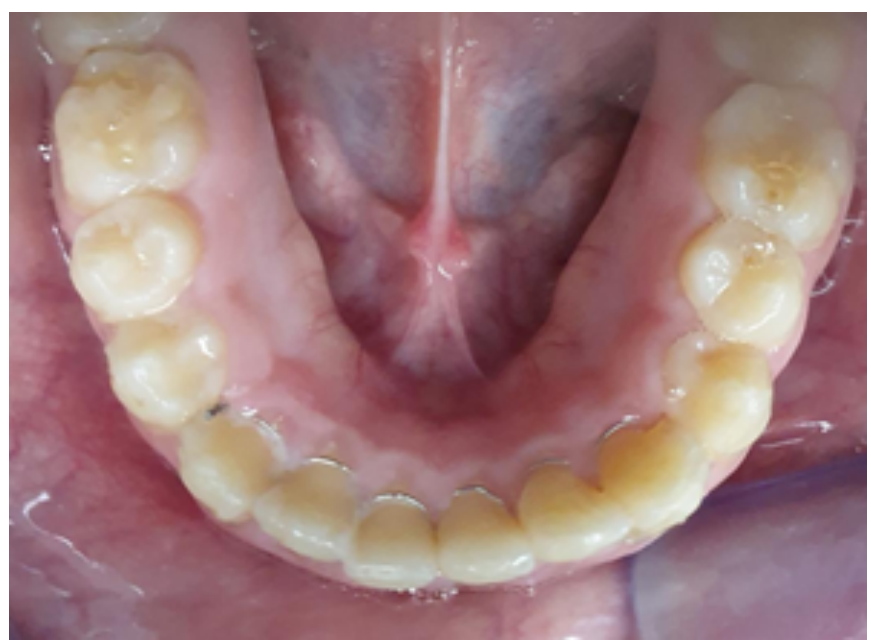

Fig. 4. Occlusal view of lower arch one month after debonding with nonbonded fixed retainer from 33 to 43 .

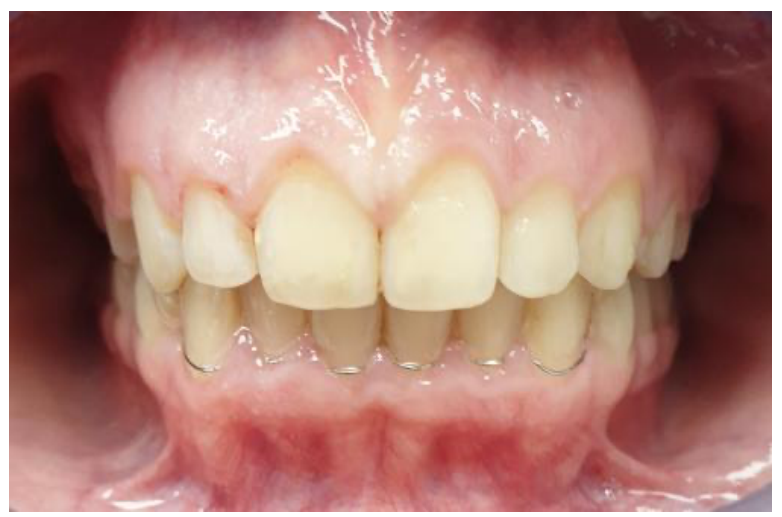

Fig. 5. Lower arch front view 4 months after debonding with non-bonded fixed figure-eight retainer.

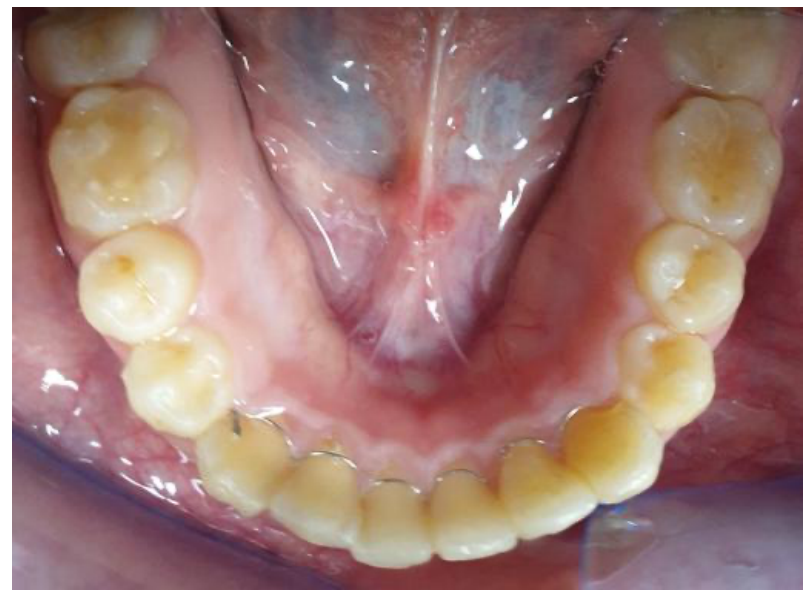

Fig. 6. Occlusal view of the lower arch 4 months after debonding with nonbonded fixed retainer from 33 to 43 .

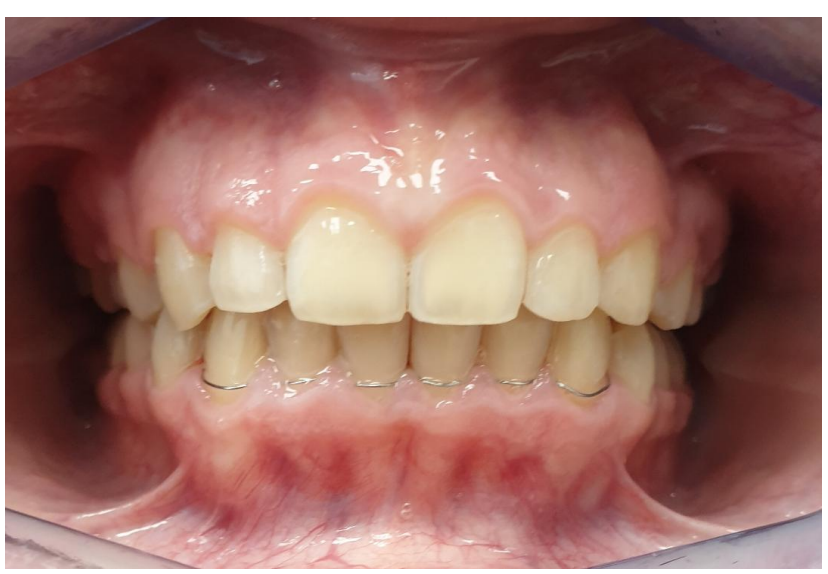

Fig. 7. Lower arch front view 6 months after debonding with non-bonded fixed retainer.

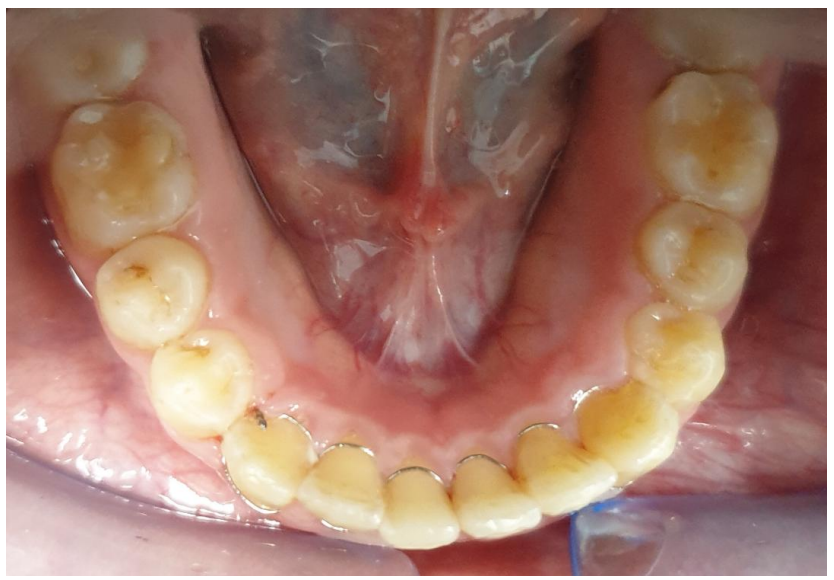

Fig. 8. Occlusal view of the lower arch 6 months after debonding with nonbonded fixed retainer from 33 to 43 . 


\section{Discussion}

The stability of treatment outcomes is contingent upon positioning teeth in the neutral zone that lies between forces arising from the tongue, lips and cheeks, a stable occlusal scheme and over-correction of rotated teeth. Factors that threaten stability include the periodontal ligament and gingival fibers of the attachment, ongoing bone growth and persistent habits. In order to counteract these and other destabilizing forces, significant effort should be devoted to ensuring effective retention [19]. Edman et al. carried out a randomized control study to evaluate the stability of treatment outcomes one-year post treatment by using three different methods of retention. Participants were divided into three groups with five participants in each group. The first group had a maxillary vacuum-formed retainer and a fixed bonded mandibular canine-to-canine retainer. The second group had a maxillary vacuum-formed retainer and underwent mandibular interproximal enamel reduction (stripping). The last group were provided with a positioner. The authors reported no significant difference between the three retention groups despite differences in age and the amount of time spent in active treatment. Furthermore, measurements made on pre, and post treatment casts revealed no significant differences amongst the three retention strategies [20].

Relapse is defined as the tendency for teeth to return to their original position. Reference [21] reported on the distinction between fast and slow relapse. Fast relapse, according to these authors, accompanies the remodeling of the periodontal ligament (PDL), whereas slow relapse is difficult to differentiate from changes that accompany growth in the craniofacial skeleton with ageing. They concluded that $25 \%$ of incisor relapse is due to natural growth and not due to relapsed orthodontic treatment. In addition, they advocated the use of a displacement index in combination with other variables to assist in differentiating between relapse attributable to orthodontic treatment versus that arising from growth changes [21].

Permanent retainers consist of a solid or braided wire adapted to conform to the newly aligned teeth. The wire is bonded onto the lingual surfaces of lower or upper incisors to stabilize them and to guard against relapse, allowing for remodeling of the periodontal ligament and maturity of the investing bone in the new tooth position. For this to occur, retention needs to remain in situ for at least nine to twelve months, and in some cases permanently. Fixed retainers are indicated in cases liable to relapse and where patient compliance is deficient. Removal of the fixed bonded retainer should be carried out by an orthodontist. A drawback of fixed bonded retainers is the extra effort required on the part of the patient to control plaque effectively in the vicinity of the appliance. However, this technique is highly recommended in patients with compromised periodontium. Nevertheless, it is essential for maintaining periodontal health and ensuring a successful outcome to treatment.

This article introduces a technique that offers several advantages over the traditional bonded retainer. Most significantly, the challenge of plaque control typically associated with traditional fixed bonded retainers is greatly reduced using a non-bondable, fixed design. Maintaining oral hygiene in the presence of the non-bonded fixed retainer requires less effort and dexterity than are required in the presence of its bonded counterpart. Interproximal cleaning is achieved by unhindered flossing without the additional requirement of floss threaders or supplementary interdental cleaning aids. The retainer is easily constructed at chair side during debonding. By avoiding the need for bonding to the tooth surfaces, the inherent risk of detachment - especially during the first two months while bone and periodontal and gingival fibers re-organize is eliminated. Similarly, the avoidance of hard foods after the placement of the Non bonded fixed retainer, necessary in the case of bonded retention, is not required. The patient can enjoy a full complement of dietary intake without restrictions. However, it is recommended to use a little thicker ligature wire than the one used. This is to avoid wire breakage during supragingival scaling with ultrasonic scaler.

This design safeguards the adaptive, physiologic capacity of the teeth to move independently within their sockets. The capacity for such movement is lost when fixed bonded retention is in place. This desirable movement allows for adaptation of the teeth to forces active on their labial and lingual surfaces and promotes periodontal health. Additional caution is required during professional debridement of the dentition carried out during dental hygiene visits. The patient needs to make the dental hygienist aware of the presence of the Kobayashi ligature wire retainer in order to avoid untoward damage during dental scaling. When excessive resistance to the passage of dental floss between adjacent teeth is encountered, the patient should arrange to be seen by an Orthodontist for interproximal stripping to be carried out. This can be repeated to the degree necessary in order to achieve unimpeded passage of floss between the teeth. This will also mitigate the tendency for relapse by consolidating the neutral zone surrounding the incisor teeth. Further, during the nine months follow up; none of the patients complained and no relapse in lower incisors alignment does occur.

\section{CONCLUSION}

The novel technique of utilizing a non-bonded fixed retainer proved superior to the traditional bonded retainer in preventing relapse during the critical period spanning two to nine months after orthodontic therapy. The method as described in this article is simple to execute and economical. It makes effective plaque control in the vicinity of the retainer significantly easier. However, studies on long term stability following lengthy periods of retention are needed. These are likely to substantiate the efficacy and durability of this technique.

\section{ACKNOWLEDGEMENT}

The author would like to express his sincere appreciation to Dr. Keith AL-Pine for his help and support in editing the manuscript and Prof Anil Sukumaran for preparing the final temple.

\section{CONFLICT OF INTEREST}

Authors declare that they do not have any conflict of interest. 


\section{REFERENCES}

[1] Bearn DR. Bonded orthodontic retainers: a review. Am J Orthod Dentofacial Orthop 1995; 108: 207-213.

[2] Liou EJ, Chen LI, Huang CS. Nickel-titanium mandibular bonded lingual 3-3 retainer: for permanent retention and solving relapse of mandibular anterior crowding. Am J Orthod Dentofacial Orthop. 2001; 119: 443-449.

[3] Zachrisson BU. The bonded lingual retainer and multiple spacing of anterior teeth. Swed Dent J Suppl. 1982; 15: 247-255.

[4] Lee RT. The lower incisor bonded retainer in clinical practice: a threeyear study. Br J Orthod. 1981; 8: 15-18.

[5] Tweed $\mathrm{CH}$. Indications for the extraction of teeth in orthodontic procedure. Am J Orthod Oral Surg. 1944; 42: 22-45.

[6] Mills JR. The long-term results of the proclination of lower incisors. $\mathrm{Br}$ Dent J. 1966; 120: 355-363.

[7] Mills JR. The stability of the lower labial segment. A cephalometric survey. Dent Pract Dent Rec. 1968; 18: 293-306.

[8] Williams R. The diagnostic line. American Journal of Orthodontics. 1969; 55: 458-476.

[9] Houston WJB, Edler R. Long-term stability of the lower labial segment relative to the A-Pog line. European Journal of Orthodontics. 1990; 12: 302-310.

[10] Schulhof RJ, Allen RW, Walters RD, Dreskin M. The mandibular dental arch: Part I, lower incisor position. Angle Orthod. 1977; 47: 280287.

[11] Shields TE, Little RM, Chapko MK. Stability and relapse of mandibular anterior alignment: a cephalometric appraisal of firstpremolar-extraction cases treated by traditional edgewise orthodontics. Am J Orthod. 1985; 87: 27-38.

[12] Zachrisson BU. Clinical experience with direct-bonded orthodontic retainers. Am J Orthod. 1977; 71: 440-448

[13] Artun J, Zachrisson B. Improving the handling properties of a composite resin for direct bonding. Am J Orthod. 1982; 81: 269-276.

[14] Zachrisson BU. Multistranded wire bonded retainers: from start to success. Am J Orthod Dentofacial Orthop. 2015; 148: 724-727.

[15] al-Emran S, Hashim H. A chairside bonded lingual retainer. J Clin Orthod. 1997; 31: 358-360.

[16] Booth FA, Edelman JM, Proffit WR. Twenty-year follow-up of patients with permanently bonded mandibular canine-to-canine retainers. Am J Orthod Dentofacial Orthop. 2008; 133: 70-76.

[17] Sachdeva RC. SureSmile technology in a patient--centered orthodontic practice. J Clin Orthod. 2001; 35: 245-253.

[18] Kravitz ND, Grauer D, Schumacher P, Jo YM. Memotain: A CAD/CAM nickel-titanium lingual retainer. Am J Orthod Dentofacial Orthop. 2017; 151: 812-815.

[19] Maltha J, Von den Hoff J, Katsaros C, Eliades T. Biological basis for orthodontic relapse. Stability, Retention and Relapse in Orthodontics. Quintessence Publishing, Berlin 2018: 16.

[20] Edman Tynelius G, Bondemark L, Lilja-Karlander E. Evaluation of orthodontic treatment after 1 year of retention--a randomized controlled trial. Eur J Orthod 2010; 32: 542-547.

[21] Abdulraheem S, Schütz-Fransson U, Bjerklin K. Teeth movement 12 years after orthodontic treatment with and without retainer: relapse or usual changes? Eur J Orthod. 2020; 42: 52-59.

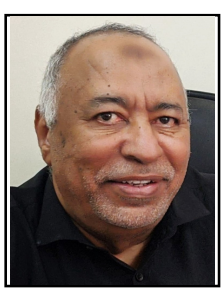

H. A. Hashim is Professor and Senior Consultant at Division of Orthodontic Hamad Medical Corporation, Doha, Qatar. 